\title{
Current Perspective in the Management of Mandibular Fractures
}

\author{
${ }^{1}$ Sachin Rai, ${ }^{2}$ Vidya Rattan
}

\begin{abstract}
The management of mandibular fractures has evolved tremendously in the last few decades. Open reduction and internal fixation with malleable monocortical titanium plates is the standard technique worldwide. The paper reviews the current concept of mandibular fracture and discusses various method and modification for different sites and clinical situations. It also covers the treatment of pediatric and geriatric mandibular fractures.
\end{abstract}

Keywords: Mandible, Jaw fracture, Open reduction, Internal fixation.

How to cite this article: Rai S, Rattan V. Current Perspective in the Management of Mandibular Fractures. J Postgrad Med Edu Res 2014;48(2):63-67.

Source of support: Nil

Conflict of interest: None

INTRODUCTION

The management of mandibular fractures has evolved tremendously in the last few decades. The conservative closed reduction techniques which were cumbersome for the surgeon and uncomfortable for the patient has given way to functionally rigid internal fixation. A brief period of bicortical extraoral fixation and compression plating three decades ago has slowly given way to the simpler monocortical fixation which can be done intraorally. The Champy's principle of osteosynthesis has stood the test of time and has proven to be the guiding line to establish monocortical fixation the worldwide choice for mandibular fracture management. ${ }^{1}$ The mandible can be divided anatomically into the horse-shoe shaped body and the vertically ascending ramus. The body has a central parasymphysis in-between the two mental foramen bilaterally. Proximal to the mental foramen, it is the body and the angle of the mandible. Each defined area has a unique anatomical variation due to the presence

\footnotetext{
${ }^{1}$ Assistant Professor, ${ }^{2}$ Additional Professor

1,2Unit of Oral and Maxillofacial Surgery, Oral Health Sciences Centre, Postgraduate Institute of Medical Education and Research, Chandigarh, India

Corresponding Author: Sachin Rai, Assistant Professor Unit of Oral and Maxillofacial Surgery, Oral Health Sciences Centre, Postgraduate Institute of Medical Education and Research, Chandigarh, India, Phone: 2756261, e-mail: drraisachin@gmail.com
}

of teeth and the inferior alveolar canal. Hence, each area is described individually.

\section{Symphysis and Parasymphysis Fractures}

Anterior mandibular fractures may or may not occur with a uni- or bilateral condylar fractures. Every possible clinical judgment should ensure open reduction and internal fixation of the anterior mandible unless contraindicated. The absence of any major vessel or nerve in this area and easy accessibility intraorally makes this zone the easiest to apply rigid fixation. Closed reduction should be preferred only if there is a hairline undisplaced fracture detected radiologically. There have been reports where the fracture has gone into nonunion when managed conservatively. ${ }^{2}$ The reason could possibly lie in the fact that this area has mainly a centripetal and less of centrifugal blood supply. Following the Champy's principle of osteosynthesis, two miniplates are sufficient to counterbalance the additional torsional forces acting on this zone ${ }^{3}$ (Figs 1A and B). With an arch bar placed superiorly, a single miniplate at the inferior border is also an acceptable modification. ${ }^{4}$ The potential damage to root apices of the anterior teeth is prevented when the superior plate is not applied. Lag screws have an advantage of providing rigid fixation and compression of the fracture segments. The proponents of this technique quote less pain, swelling and early return of function as the major advantages of lag screw fixation. ${ }^{5}$ The method is technique sensitive and has a slow learning curve. At times with more laterally extending fractures, the plate may extend up to the formen. A carful dissection of the nerve branches and lifting it superiorly may help the plate to slide below the foramen and ease in drilling and applying screws.

\section{Body Fracture}

The body of the mandible is less in height compared to the anterior mandible. The superior half has roots of teeth below the buccal cortex and approximately in the middle runs the inferior alveolar canal. So clinically, the only possibility of applying a plate is at the inferior border. An arch bar placed superiorly prevents distraction of the fracture segments and the lower plate utilizes the natural compression forces at the lower border to provide functionally rigid fixation (Figs 2A and $\mathrm{B}$ ). In absence of an arch bar or missing teeth, a small two-hole plate can act as a tension band superiorly. 


\section{Angle Fracture}

Angle is one of the most widely debated anatomical sites in context of nature of fixation in mandible. ${ }^{6}$ The complex three-dimensional (3D) anatomy, twin muscle attachment and the presence of third molar in various stage of eruption makes this area unique and controversial. Complication related to open reduction and internal fixation is the highest reported in the maxillofacial literature. Proponents have argued and put forward various treatment modalities with their advantages and disadvantages. Closed reduction, open reduction with single miniplate or bicortical fixation with reconstruction plate are the current treatment options. The most favored and accepted is the fixation of angle fracture by a single miniplate at the superior border following the Champy's line of osteosynthesis ${ }^{7}$ (see Figs 2A and B). The third molar in the line of fracture should be preferably removed prior to fixation. The authors do not find any possible advantage of preserving it. ${ }^{8}$ Removing it enables primary closer and makes it easy to maintain hygiene.

\section{Pediatric Fractures}

Mandibular fractures in children pose a different challenge to the maxillofacial surgeon. The presence of tooth buds and an eminent danger to damage the inferior alveolar neurovascular bundle in a thin mandible makes open reduction and internal fixation a relative contraindication. Closed reduction with acrylic splints and circum-mandibular wiring is a favored technique for managing these cases. ${ }^{9}$ At times, a situation arises where the reduction is not possible with this technique. Displaced or multiple fracture segments or infected old fractures make it mandatory to open and reduce the segments. Fixation should be done at the inferior border and with thin plates and monocortical screws ${ }^{10}$ (Figs 3A and B). Bioresorbable plates and screws have been advocated and accepted for pediatric jaw fractures. The resorption of implants after a specific period does not interfere in growth and also does not pose a hindrance in radiological intervention later on in life. ${ }^{11}$ The period of jaw fixation with elastics or wires should be approximately 2 weeks as healing is much rapid in case of children.

\section{Geriatric Fractures}

Mandibular fractures in elderly pose a completely opposite set of problems compared to children and young adults. Decreased jaw height due to progressive loss of tooth, decreased centrifugal or endovascular blood supply and decreased bone density makes the mandible weak and prone to fracture. In addition to the local factors, presence of systemic illness (e.g. diabetes mellitus) may or may not disturb the healing of bone. Various cardiopulmonary

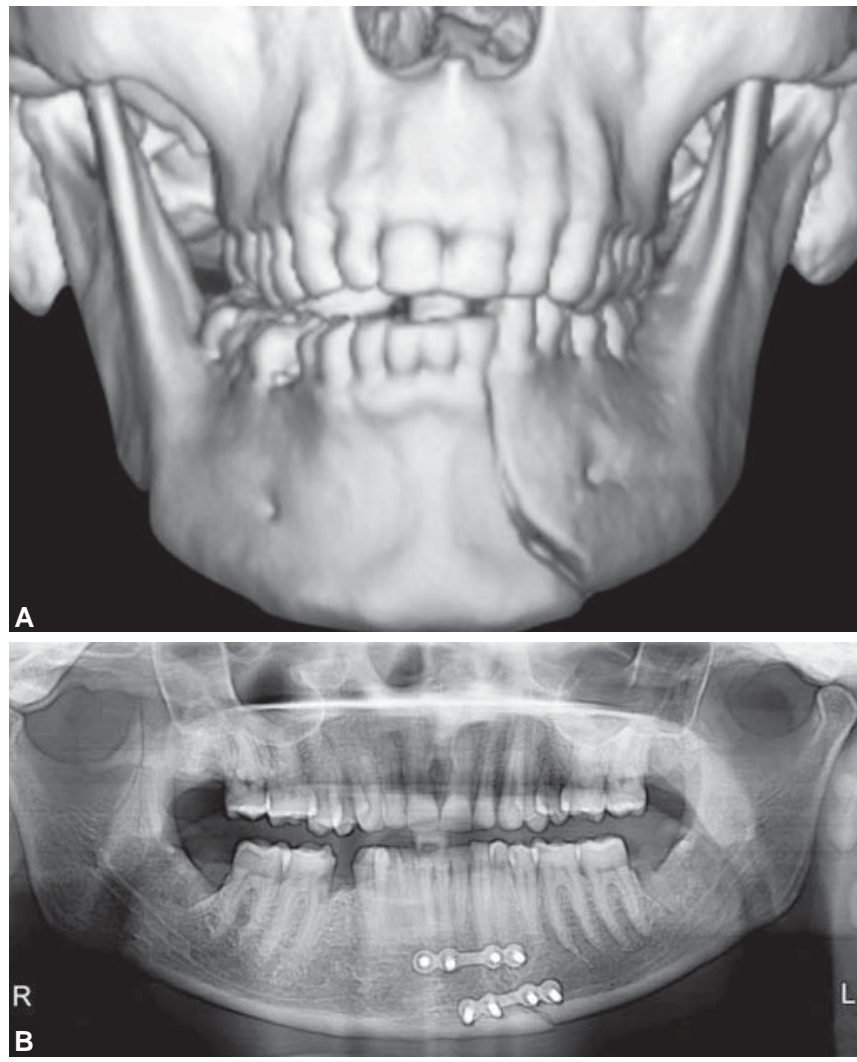

Figs $1 \mathrm{~A}$ and $\mathrm{B}$ : An anterior mandibular fracture managed with two miniplates
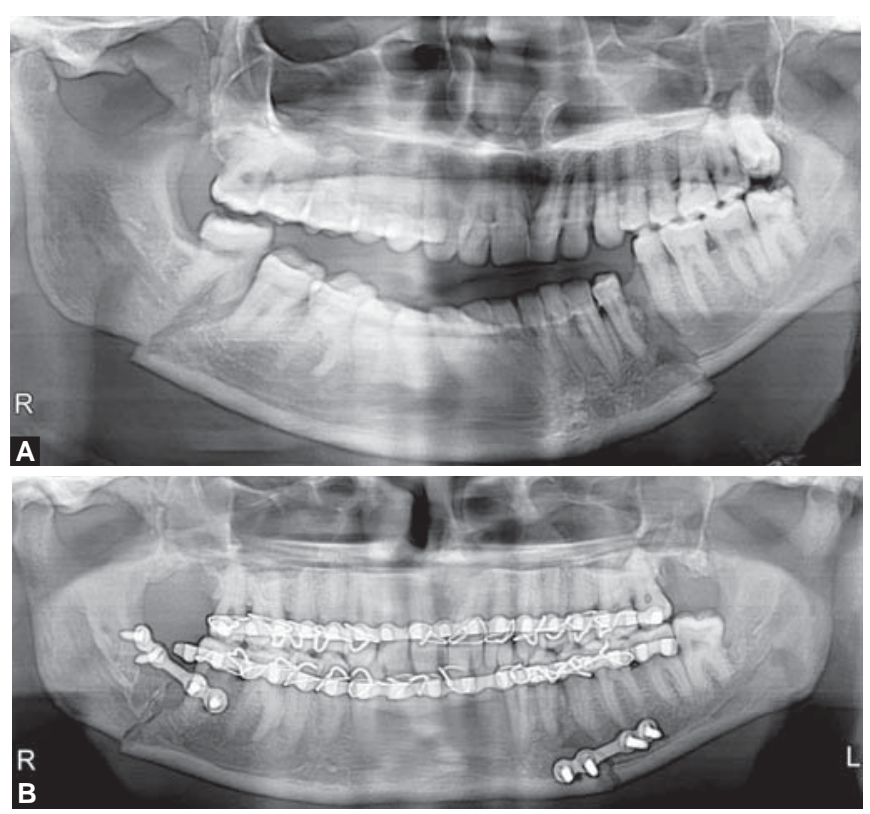

Figs 2A and B: Fixation of a bilateral fracture mandible (body and angle) with single miniplates each

ailments may worsen the condition and pose as a relative contraindication for any surgical intervention under general anesthesia. The current accepted concept in geriatric mandible fracture is to go for open reduction and internal fixation. It has been shown that the healing and return of function is much better and swift compared to using closed nonrigid fixation techniques. ${ }^{12}$ Closed reduction with jaw fixation is cumbersome and less tolerable to the elderly. 
It also drastically reduces the vital capacity of the lungs and may aggravate respiratory problems. ${ }^{12}$ At times, due to absence or poor health of the teeth, it is ineffective to apply arch bars and the nonrigid fixation in these situations may progress the fracture into nonunion. If general anesthesia is not a relative contraindication for such patients, the maxillofacial surgeon should prefer rigid fixation. Depending upon the local factors like displacement, presence of infection or old fractures, the surgeon can choose between miniplate and reconstruction plates. A typical common fracture in this group of patients is a bilateral body fracture or bucket-handle fracture (Figs 4A and B).

\section{Fractures occurring in Combination}

Although the site of fracture in the mandible depends on the nature and the direction of the force, there are certain bilateral fractures which are seen more commonly as combination fractures. The arched horse-shoe shaped body and the vertical ramus create some unique buttresses and some weak sites which are more prone to fracture with direct and indirect forces. Common fractures occurring in combinations are anterior mandible and uni- or bilateral condyles. These are commonly called as guardsman fracture or parade ground fractures. Another combination seen more often is angle and condyle fracture or angle and body fracture (see Figs 2A and B). The neck of the condyle been the weakest area in the mandible is seen commonly in combination fractures. In such bilateral situations, the objective should be to fix atleast one site with rigid fixation. The second site can be managed with a functionally stable fixation or semi-rigid fixation. ${ }^{13}$ The condyles can be managed with closed reduction and the body fracture can be managed with a rigid fixation. The sequencing in multiple fractures varies. The most favored way of approaching them is to do the easier fractures first which is mostly the anterior body of mandible. The authors too propose the same and advocate opening the entire fractures site, reducing the segments and achieving occlusion followed by rigid fixation. Similarly in sequencing of panfacial fractures, the mandible is ahead in the algorithm. A rigid fixation of fracture mandible provides adequate guidance for occlusion and a stable base to build the upper face. Also fixing the condyles restores the vertical dimension of the lower face. ${ }^{14}$

\section{Comminuted Fractures}

Comminution refers to the condition of multiple lines of fracture within the same region of the mandible. Comminuted fractures are less common than linear fractures but can occur at any location throughout the mandible. Such injuries require load-bearing fixation because the bony fragments are not capable of sharing any of the functional loads transmitted to the bone fragments. Load-bearing fixation in any region of the mandible is provided by a reconstruction bone plate, using a minimum of three screws on each side of the comminuted area. The comminuted area is simply bridged by the bone plate. To facilitate treatment of comminuted fractures, one should first 'simplify' the fracture. ${ }^{13}$ After the occlusion has been reestablished using intermaxillary fixation, the fracture is exposed and wires or small plates are used to hold the fragments into their proper relationship
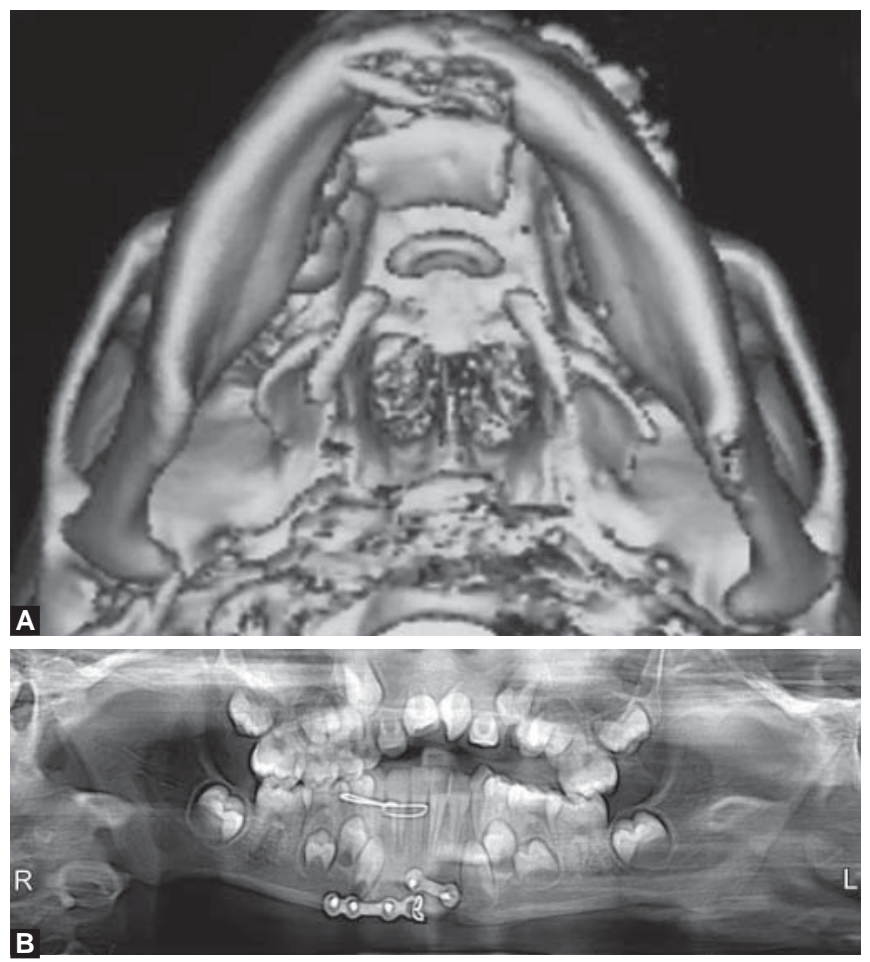

Figs $3 \mathrm{~A}$ and B: A displaced pediatric mandibular fracture managed with miniplates and wires

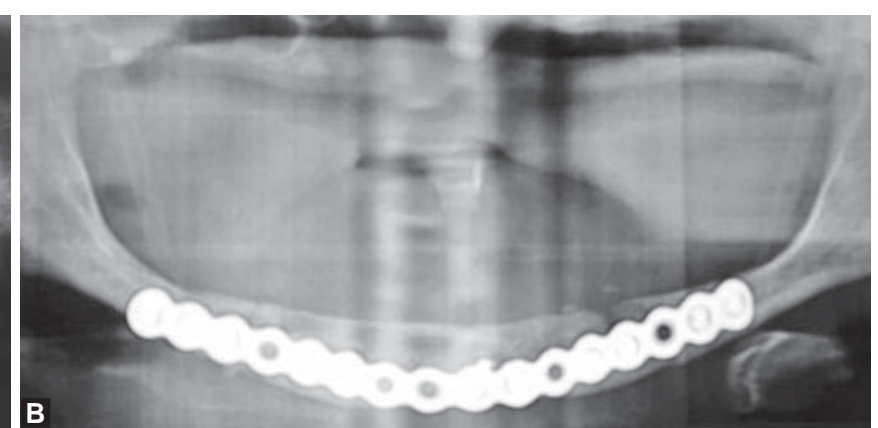

Figs 4A and B: An edentulous bilateral fracture mandible in a geriatric patient managed with a load bearing reconstruction plate 

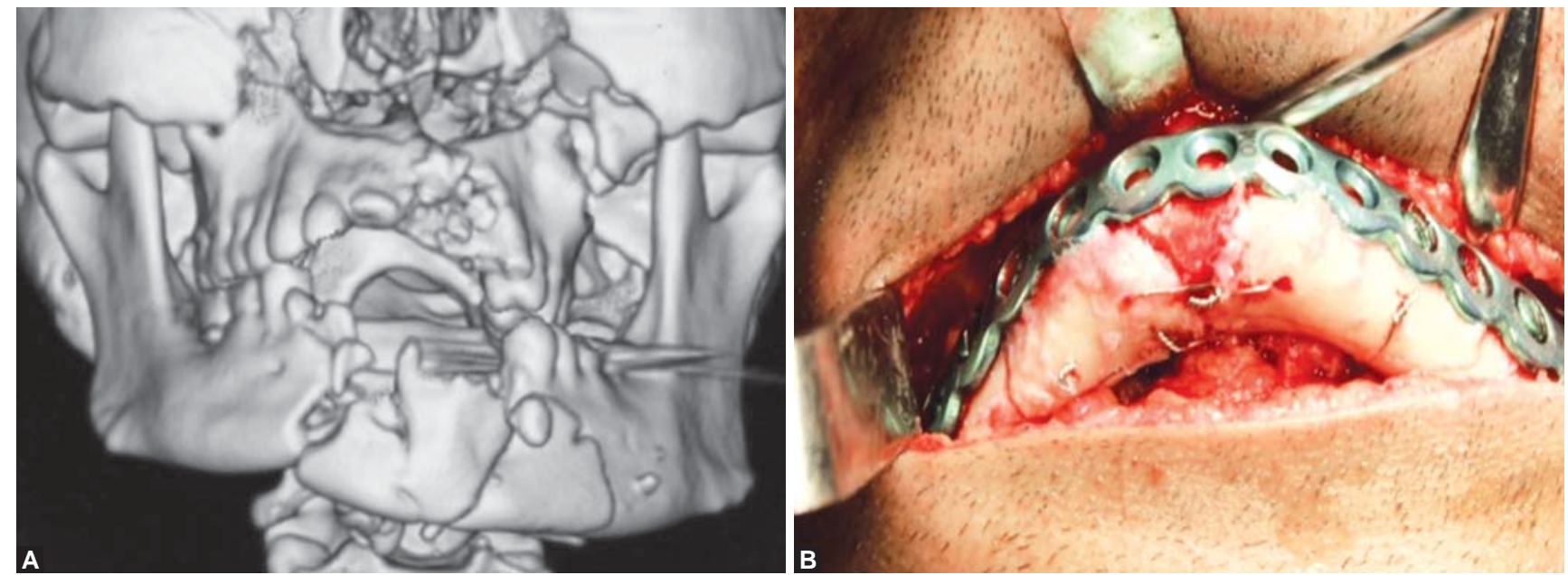

Figs 5A and B: A comminuted anterior mandibular fracture managed with wire fixation to align the small fragments followed by rigid fixation with reconstruction plate

to one another. A reconstruction bone plate is then applied to bridge the area of comminution (Figs 5A and B).

\section{DISCUSSION}

The objective of treating any mandibular fracture is to restore symmetry and occlusion and also to restore adequate movement and joint function. Definitive repair is not a surgical emergency and it can wait in a patient with multiple injuries. A temporary reduction and stabilization with an arch bar maybe encouraged in such patients. Early elastic intermaxillary fixation helps in reduction of displaced segments and thus pain and inflammation. Closed reduction should be reserved for undisplaced fractures and where somehow open reduction is a relative contraindication. A lot of emphasis has been laid on wireless fixation techniques to minimize needle stick injuries. Hand-held reduction, intermaxillary (IMF) screws and rapid $\mathrm{IMF}^{\mathrm{TM}}$ are alternative methods to stabilize the jaw temporarily. ${ }^{15}$

The management of mandibular fractures has come a long way from barrel bandage application and extraoral pin fixation. Spiessl et al (1970) studied and established AO/ ASIF principles and propagated compression plating. ${ }^{16}$ The AO group advocated absolute rigid fixation as the ideal treatment modality. The technique sensitive armamentarium and bulky plates applied by extraoral route could not gain much popularity as expected by its propagators. Brons and Boering (1970) introduced lag screw technique. ${ }^{17}$ This technique too was technique sensitive and resulted in compression and absolute fixation. The concept of absolute rigid fixation gave way to functionally rigid fixation achieved by the small monocortical plates and screws. Michelet et al (1973) used bendable, monocortical miniplates based on the Champy's principle of osteosynthesis for fixation of mandibular fractures. ${ }^{18}$ It laid the foundation of open reduction and internal fixation. The ease of applicability and functionally acceptable results has made it the preferable method of fracture management.
In the consecutive decades, further research went on regarding the material and design of the implants. Threedimensional plates, trapezoidal plates and plates with screw locking design have been accepted and clinically used. The locking plate transfers the mandibular forces to itself without compressing the bone beneath it. Thus, the screw plate system acts as one unit. Bioresorbable plates have been advocated but due to their inefficiency to provide rigid fixation they have not gained much popularity among maxillofacial surgeons. Their application is still limited to pediatric fractures.

Tooth in the line of fracture has been a point of debate for a long period. The general consensus now is to preserve the tooth which can help in anatomical reduction of the fracture segments. The absolute indications to remove tooth in the fracture line are: (i) vertical fracture, (ii) non-restorable caries with/without periapical pathology, (iii) malpositioned tooth hindering in restoration of occlusion and (iv) any third molar in the line of fracture. ${ }^{8}$

\section{CONCLUSION}

We propose that early and painfree mobilization should be the aim of all mandibular fracture treatment. Except for undisplaced fractures, open reduction and internal fixation should be the method of choice for every case unless contraindicated. Tooth in the line of fracture, except of third molars, should be preserved. Monocortical fixation should be encouraged due to better patient compliance, ease of applicability and less technique sensitivity.

\section{REFERENCES}

1. Champy M, Loddé JP, Schmitt R, Jaeger JH, Muster D. Mandibular osteosynthesis by miniature screwed plates via a buccal approach. J Maxillofac Surg 1978;6:14-21.

2. Mathog RH, Toma V, Clayman L, Wolf S. Nonunion of the mandible: an analysis of contributing factors. J Oral Maxillofac Surg 2000;58:746-752. 
3. Kroon FH, Mathisson M, Cordey JR, Rahn BA. The use of miniplates in mandibular fractures: An in vitro study. J Craniomaxillofac Surg 1991;19:199-201.

4. Ellis E. Astudy of two bone plating methods for fractures of the mandibular symphysis/body. J Oral Maxillofac Surg 2011;69:19781987.

5. Ellis E. Is lag screw fixation superior to plate fixation to treat fractures of the mandibular symphysis? J Oral Maxillofa Surg 2012; 70:875-882.

6. Ellis E, Walker L. Treatment of mandibular angle fractures using two non-compression miniplates. J Oral Maxillofac Surg 1994;52: 1032-1034.

7. Ellis E, Walker LR. Treatment of mandibular angle fractures using one non-compression miniplate. J Oral Maxillofac Surg 1996; 54:864-867.

8. Rai S, Pradhan R. Tooth in the line of fracture: its prognosis and its effects on healing. Indian J Dent Res 2011;22:495-496.

9. Patrick CP, Kaufman Y, Izaddoost S, DA Hatef, Hollier L. Principles of pediatric mandibular fracture management. Plast Reconstr Surg 2009;123:1022-1024.

10. Smartt JM, Jlow DW, Bartlett SP. The pediatric mandible: II. Management of traumatic injury or fracture. Plast Reconstr Surg 2005;116:28.
11. Eppley BL. A resorbable and rapid method for maxillomandibular fixation in pediatric mandible fractures. J Craniofac Surg 2000; 11:236.

12. Goldschmidt MJ, Castiglione CL, Assael LA. Craniomaxillofacial trauma in the elderly. J Oral Maxillofac Surg 1995;53:11451149.

13. Ellis E, Miles BA. Fractures of the mandible: a technical perspective. Plast Reconstr Surg 2007;120(Suppl 2):76.

14. Kelly KJ, Manson PN, Vander Kolk CA, Markowitz BL, Dunham CM, Rumley TO, Crawley WA. Sequencing LeFort fracture treatment (organization of treatment for a panfacial fracture). J Craniofac Surg 1990;1:168-178.

15. Cousin GC. Wire-free fixation of jaw fractures. Br J Oral Maxillofac Surg 2009;4:521-524.

16. Strelzow VV, Friedman WH. Dynamic compression plating in the treatment of mandibular fractures. Early experience. Arch Otolaryngol 1982;108:583-586.

17. Brons R, Boering G. Fractures of the mandibular body treated by stable internal fixation: a preliminary report. J Oral Surg 1970; 28:407-415.

18. MicheletA, Deymes J. Osteosynthesis with screwed plates in maxillofacial surgery. Experience with 500 satellite plates. Int Surg 1973;58:249-253. 